\title{
MGMT promoter methylation in patients with glioblastoma: is methylation-sensitive high-resolution melting superior to methylation-sensitive polymerase chain reaction assay?
}

\author{
Shinji Yamashita, MD, Kiyotaka Yokogami, MD, PhD, Fumitaka Matsumoto, MD, \\ Kiyotaka Saito, MD, Asako Mizuguchi, MD, Hajime Ohta, MD, PhD, and Hideo Takeshima, MD, PhD
}

Division of Clinical Neuroscience, Department of Neurosurgery, Faculty of Medicine, University of Miyazaki, Japan

\begin{abstract}
OBJECTIVE The methylation status of the 06-methylguanine-DNA methyltransferase (MGMT) gene promoter is a prognostic factor in adults with glioblastoma (GBM); it also yields information that is useful for clinical decision-making in elderly GBM patients. While pyrosequencing is the gold standard for the evaluation of the methylation status of MGMT, methylation-sensitive polymerase chain reaction (MS-PCR) assay continues to be used widely. Although MS-PCR results exhibited a good correlation with the prognosis of patients with GBM treated under the Stupp protocol, interpretation of the bands is based on subjective judgment, and the assay cannot be used to analyze heterogeneously methylated samples. We assessed whether methylation-sensitive high-resolution melting (MS-HRM) is an alternative to MS-PCR.
\end{abstract}

METHODS The authors prepared 3 primer sets that covered CpG 72-89 for MS-HRM analysis to determine the methylation levels of 6 human glioma cell lines. The results were validated by bisulfite sequencing of cloned alleles. The authors also subjected surgical samples from 75 GBM patients treated with temozolomide (TMZ) to MS-HRM to assess the MGMT methylation status and compared the findings with MS-PCR results using receiver operating characteristic (ROC), univariate, and multivariate analyses.

RESULTS There was a strong correlation between the methylation levels of the 6 glioma cell lines evaluated by MSHRM and by bisulfite sequencing; with primers 1 and 2 , the correlation was significant $(r=0.959$ and $r=0.960$, respectively, $p<0.01$ ). Based on log-rank analysis, MS-HRM was significantly better than MS-PCR for predicting progressionfree survival (PFS) and overall survival (OS) based on the methylation status of the MGMT promoter (PFS predicted by MS-HRM and MS-PCR $=0.00023$ and 0.0035 , respectively; $O S=0.00019$ and 0.00028 , respectively). $R O C$ analysis showed that the area under the curve was larger with MS-HRM than with MS-PCR (PFS: 0.723 vs 0.635 ; OS: 0.716 vs 0.695). Based on multivariate Cox regression analysis, MS-HRM was significantly better than MS-PCR for predicting the treatment outcome (MS-HRM vs MS-PCR: PFS, $p=0.001$ vs 0.207 ; OS, $p=0.013$ vs 0.135 ).

CONCLUSIONS The authors' findings show that MS-HRM is superior to MS-PCR for the detection of MGMT promoter methylation. They suggest MS-HRM as an alternative to MS-PCR for assessing the prognosis of patients with GBM.

https://thejns.org/doi/abs/10.3171/2017.11.JNS171710

KEYWORDS 06-methylguanine-DNA methyltransferase; MGMT; methylation-sensitive high-resolution melting; methylation-sensitive polymerase chain reaction; temozolomide; glioblastoma; oncology

$\mathrm{G}$ LIOMAS account for $70 \%$ of human malignant primary brain tumors, and their prognosis is poor. ${ }^{14}$ Patients diagnosed with glioblastoma (GBM), the most malignant type of glioma (WHO grade IV), survive for an average of 14.6 months, even with the current stand- ard treatment that includes surgery followed by the combined use of radiotherapy and temozolomide (TMZ). ${ }^{16,17}$ TMZ increases the level of O6-methylguanine and thereby elicits DNA damage-induced apoptosis. ${ }^{8}$ The cytotoxic effects of TMZ are weakened by O6-methylguanine-DNA

\footnotetext{
ABBREVIATIONS AUC = area under the curve; GBM = glioblastoma; KPS = Karnofsky Performance Status; $M G M T=06$-methylguanine-DNA methyltransferase; MS-HRM $=$ methylation-sensitive high-resolution melting; $M S-P C R=$ methylation-sensitive polymerase chain reaction; $O S=$ overall survival; $P F S=$ progression-free survival; ROC = receiver operating characteristic; $\mathrm{TMZ}=$ temozolomide.
}

SUBMITTED July 24, 2017. ACCEPTED November 10, 2017.

INCLUDE WHEN CITING Published online May 4, 2018; DOI: 10.3171/2017.11.JNS171710. 
methyltransferase (MGMT), a suicide-repair enzyme that reverses DNA damage by removing alkylating adducts from guanines. ${ }^{8,22}$ Because $M G M T$ gene expression is highly regulated at the epigenetic level via $\mathrm{CpG}$ islandpromoter methylation, the $M G M T$ methylation status can help to predict the effectiveness of TMZ treatment. ${ }^{1,2}$

Methylation-sensitive polymerase chain reaction (MSPCR) assay has been used to detect the MGMT methylation status. Although a good correlation between the methylation status and GBM prognosis has been reported, MS-PCR results are evaluated subjectively based on the interpretation of electrophoretic bands. Also, the $\mathrm{CpG}$ island of MGMT is heterogeneously methylated, and heterogeneously methylated samples cannot be assayed by MS-PCR. ${ }^{9,12,13,23}$ Since MS-PCR primers are designed to specifically amplify only fully methylated sequences in the primer region, the effect of heterogeneous methylation on the results is unpredictable. ${ }^{3,11}$ Furthermore, not all commonly used MS-PCR primers cover the region crucial for silencing $M G M T$ transcription. The human MGMT gene is reported to harbor a 762-base-pair $\mathrm{CpG}$ island that contains $98 \mathrm{CpG}$ sites in the promoter region $(-531$ to +231 from ATG). According to Malley et al., ${ }^{9} \mathrm{CpG} 73-90$ is the region critically involved in transcription. Although most commonly used primers for MS-PCR target this region (forward: $\mathrm{CpG} 76-80$; reverse: $\mathrm{CpG} 84-87$ ), not all $\mathrm{CpG}$ sites of interest can be evaluated. ${ }^{4}$ Consequently, MS-PCR is not the ideal method for assessing the $M G M T$ methylation status of the MGMT promoter.

To overcome the limitations of MS-PCR, Quillien et al. ${ }^{13}$ proposed pyrosequencing. Although it is a robust and accurate method for detecting the MGMT methylation status, it is expensive and requires special instrumentation not available in all laboratories. Therefore, we examined whether methylation-sensitive high-resolution melting (MS-HRM) is an alternative to MS-PCR. MS-HRM is based on the principle that the melting temperature of a PCR product depends on the methylation status of the locus at which the PCR product originates. Therefore, the methylation status of loci of interest can be quantified by comparing the melting profiles of unknown PCR products with known standard samples. As MS-HRM analysis is less expensive, faster, and less laborious than pyrosequencing, ${ }^{21}$ we examined its potential as an alternative for the detection of the methylation status of the MGMT promoter.

To test the reliability of MS-HRM for the quantitative assessment of the MGMT methylation level, we used 6 human glioma cell lines. The results were validated by bisulfite sequencing of cloned alleles, the gold standard for quantitatively evaluating methylation levels. Next, we obtained the methylation level of tumor samples from 75 GBM patients treated with TMZ and compared MS-HRM and MS-PCR findings using receiver operating characteristic (ROC), univariate, and multivariate analyses. Here, we report our results and discuss factors underlying the difference between MS-HRM and MS-PCR findings.

\section{Methods}

\section{Patients and Tumor Samples}

Our study was approved by the institutional review board of Miyazaki University Hospital, Japan. All patients gave their prior written informed consent for the use of their tissues in this study. We included 75 newly diagnosed GBM patients who had undergone surgery at our institution between February 2008 and July 2015. They received basically standard-care treatment consisting of the concomitant delivery of TMZ and irradiation (Stupp proto$\mathrm{col})$. The general characteristics of the patients, including their age, sex, Karnofsky Performance Status (KPS), and the extent of resection, were obtained from their medical records and are shown in Table 1. The median followup was 17 months; the median progression-free survival (PFS) was 9 months (95\% CI 6.0-11.1 months), and the median overall survival (OS) was 17 months (95\% 13.025.1 months).

Tissue specimens $(n=75)$ obtained at surgery were divided into 2 parts: one part was examined histologically, and the other was frozen in liquid nitrogen and stored at $-80^{\circ} \mathrm{C}$ until DNA extraction.

\section{Cell Culture}

The established glioma cell lines (LN18, LN229, U87MG, U251, A172, and T98G) were a gift from the Department of Pathology of the University of Miyazaki. LN18 and LN229 were incubated in DMEM+L-glutamax supplemented with $5 \%$ fetal bovine serum and $1 \%$ penicillin/streptomycin $\left(37^{\circ} \mathrm{C}, 5 \% \mathrm{CO}_{2}\right)$. The other cell lines were grown in DMEM supplemented with $10 \%$ fetal bovine serum and $1 \%$ penicillin/streptomycin $\left(37^{\circ} \mathrm{C}, 5 \% \mathrm{CO}_{2}\right)$.

\section{DNA Extraction and Bisulfite Treatment}

Genomic DNA was isolated from the cell lines and the frozen surgical specimens using the QIAamp DNA Mini Kit (Qiagen) according to the manufacturer's instructions. The DNA quantity was measured using NanoDrop 2000 (Thermo Fisher Scientific). Genomic DNA (1 $\mu \mathrm{g})$ was bisulfite-treated using the EpiTect (Qiagen) or the Innu CONVERT bisulfite basic kit (Analytik Jena AG) according to the manufacturer's instructions to convert all unmethylated cytosine to uracil, leaving 5-methylcytosine unaltered. The DNA methylation status of the MGMT promoter was analyzed by MS-PCR and MS-HRM.

\section{Analysis of MGMT Promoter Methylation by MS-PCR}

Converted DNA was subjected to MS-PCR using 2 primer pairs designed for the amplification of methylated and unmethylated alleles of the $M G M T$ promoter. The primer sequence for unmethylated reactions was $5^{\prime}$ TTTGTGTTTTGATGTTTGTAGGTTTTTGT-3' (forward), 5'-AАСТCCACACTCTTCCAAAAACAAAA CA-3' (reverse); for methylated reactions it was 5'-TTTC GACGTTCGTAGGTTTTCGC-3' (forward), 5'-GCACT CTTCCGAAAACGAAACG-3' (reverse). ${ }^{5}$ PCR was performed in a 2720 thermal cycler (Applied Biosystems). The PCR conditions were an initial melting step (10 minutes at $95^{\circ} \mathrm{C}$ ); 30 cycles of 20 seconds at $95^{\circ} \mathrm{C}, 20$ seconds at $60^{\circ} \mathrm{C}$, and 45 seconds at $72^{\circ} \mathrm{C}$; and a final elongation $(4$ minutes at $72^{\circ} \mathrm{C}$ ). Amplified products were loaded on $16 \%$ polyacrylamide gels and visualized under ultraviolet light using ethidium bromide staining. 
TABLE 1. Characteristics of the 75 patients from whom the GBM samples were obtained

\begin{tabular}{cc}
\hline \multicolumn{1}{c}{ Variable } & Value \\
\hline Median age at op in yrs (range) & $64(32-84)$ \\
\hline Sex & 29 \\
\hline F & 46 \\
\hline M & \\
\hline GTpe of op & 50 \\
\hline Partial & 23 \\
\hline Biopsy & 2 \\
\hline KPS score & \\
\hline $90-100$ & 18 \\
\hline $70-80$ & 34 \\
\hline$<70$ & 23 \\
\hline IDH1 & $5(6.8)$ \\
\hline Mutated & $69(93.2)$ \\
\hline Wild type & $51(79.7)$ \\
\hline TERT & $13(20.3)$ \\
\hline Mutated &
\end{tabular}

GTR = gross-total resection.

Values are presented as the number of patients (\%) unless stated otherwise.

\section{Analysis of MGMT Promoter Methylation by MS-HRM Curve Analysis}

For sequential PCR amplification and HRM analysis, we used the StepOne system (Thermo Fisher Scientific). PCR was performed in a total volume of $20 \mu \mathrm{l}$ $(10 \mu \mathrm{l}$ of MeltDoctor HRM master mix [Applied Biosystems], $1.2 \mu \mathrm{l}$ of each primer $[10 \mu \mathrm{M}]$ ) and $20 \mathrm{ng}$ of bisulfite-converted DNA. The primer sets were 5'-GC GTTTCGGATATGTTGGGATAGT-3' (forward), 5'-C CTACAAAACCACTCGAAACTACCA-3' (reverse)

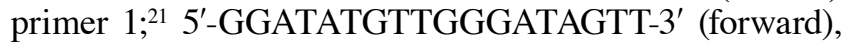
5'-CCCAAACACTCACGAAAT-3' (reverse) primer 2, ${ }^{18}$ and $5^{\prime}$-CGTTTGCGATTTGGTGAGTGTT-3' (forward), 5'-CCTACAAAACCACTCGAAACTACCA-3' (reverse) primer $3 .^{21}$

The PCR conditions were 2 minutes at $95^{\circ} \mathrm{C} ; 45$ cycles of 10 seconds at $95^{\circ} \mathrm{C}, 30$ seconds at $54^{\circ} \mathrm{C}$, and 15 seconds at $72^{\circ} \mathrm{C}$; and a final HRM step of 30 seconds at $95^{\circ} \mathrm{C}$, 60 seconds at $60^{\circ} \mathrm{C}, 10$ seconds at $70^{\circ} \mathrm{C}$, and continuous acquisition to $90^{\circ} \mathrm{C}$ at 1 acquisition per $0.2^{\circ} \mathrm{C}$. DNA extracted from normal lymphocytes was treated with $\mathrm{CpG}$ methylase M.sssI (New England Biolabs) to represent fully methylated MGMT alleles. Untreated DNA from normal lymphocytes represented fully unmethylated alleles. DNA was bisulfite-converted and mixed with fully unmethylated alleles to obtain $0 \%, 5 \%, 10 \%, 30 \%, 50 \%$, and $100 \%$ methylation standards. MS-HRM data were analyzed using HRM software (version 3.0.1, Thermo Fisher Scientific). Output plots were produced as aligned melting curves (Supplemental Fig. 1). The area under the curve (AUC) was calculated from the aligned melting curves using ImageJ (NIH); linear regression was applied to interpolate unknown samples from the standards (Supplemental Figs. 2 and 3). All measurements were performed in triplicate.

\section{Analysis of MGMT Promoter Methylation by Cloning-Based Bisulfite Sequencing}

The $3^{\prime}$ and $5^{\prime}$ sequences of PCR products derived from MS-HRM using primer 1 were digested with BamH1 and $E c o R 1$ and ligated into a pBluescript II plasmid using $2 \times$ ligation mix (Wako); subcloning-efficient DH5 $\alpha$-competent cells (Invitrogen) were used for transformation. Primer 1 was the sequencing primer. PCR products derived from 25 colonies of each sample were used for the sequencing reaction using BigDye Terminator v1.1 (Applied Biosystems) according to the manufacturer's instructions. The cycling conditions were 60 seconds at $96^{\circ} \mathrm{C}$; and 25 cycles of 30 seconds at $96^{\circ} \mathrm{C}, 5$ seconds at $50^{\circ} \mathrm{C}$, and 4 minutes at $60^{\circ} \mathrm{C}$. The PCR products were cleaned using Centri-Sep Spin Columns (Thermo Fisher Scientific) and then sequenced on an ABI PRISM 310 genetic analyzer (Applied Biosystems).

\section{Analysis of IDH1 and TERT Mutations}

IDHI mutations were confirmed by immunohistochemical analysis using either R132H antibody (1:20 dilution, H09, Dianova) or direct sequencing. The primer sequence for IDH1 was 5'-GGCTTGTGAGTGGATGGGTA-3' (forward), 5'-GCAAAATCACATTATTGCCAAC-3' (reverse). Analysis was as previously described. ${ }^{15}$

TERT mutations were confirmed by direct sequencing. A fragment of the TERT promoter was amplified with a primer (5'-GTCCTGCCCCTTCACCTT-3' [forward], 5'-GCACCTCGCGGTAGTGG-3' [reverse]) that contained the C228T and C250T mutation sites. The PCR conditions were initial denaturation at $95^{\circ} \mathrm{C}$ for $60 \mathrm{sec}-$ onds; and 35 cycles of $95^{\circ} \mathrm{C}$ denaturation for 30 seconds, $58^{\circ} \mathrm{C}$ annealing for 30 seconds, and elongation for $20 \mathrm{sec}-$ onds at $72^{\circ} \mathrm{C}$. Sequencing was performed with BigDye Terminator v1.1 on an ABI PRISM 310 genetic analyzer.

\section{Statistical Analysis}

ROC curves were produced to assess the sensitivity and specificity of MS-HRM and MS-PCR for predicting $\mathrm{PFS} \geq 10$ months and OS $\geq 18$ months. Data obtained by univariate analysis and on survival curves were analyzed with the Kaplan-Meier method and compared using the log-rank test. For multivariate analysis, we applied multiple Cox regression analysis. To evaluate the correlation between methylation levels determined by MS-HRM and by cloning-based bisulfite sequencing, we used the Pearson test. Differences of $p<0.05$ were considered statistically significant. Statistical analyses were performed with StatMate III software (ATMS) and Prism 6 (GraphPad).

\section{Results}

Glioma Cell Lines: Region of Interest in the MGMT Promoter and MS-HRM Analysis

The promoter region of the $M G M T$ gene harbors 98 

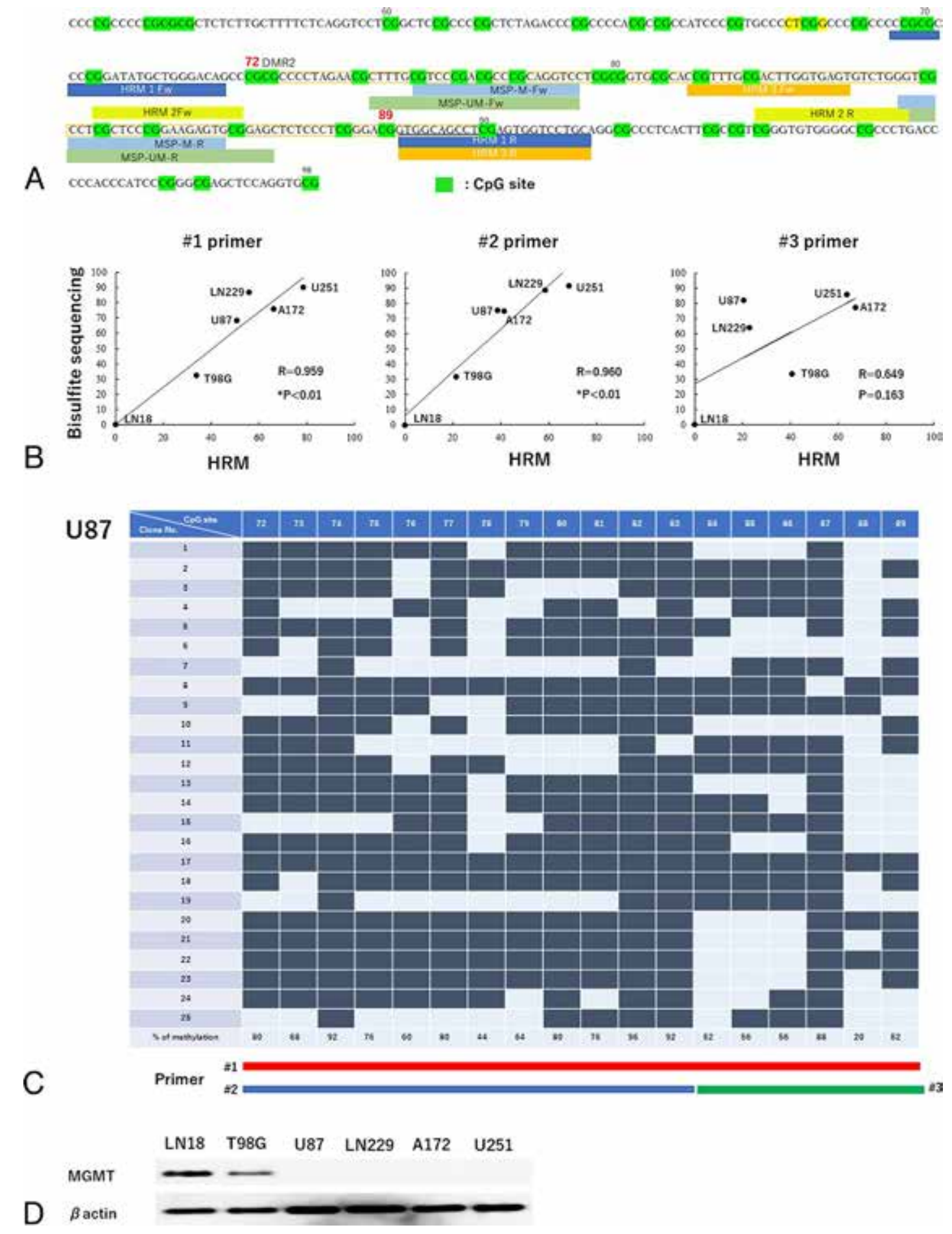

FIG. 1. Glioma cell cultures. Region of interest in the MGMT promoter and MS-HRM analysis of glioma cell lines. A: MGMT promoter methylation was analyzed by MS-HRM and MS-PCR. For MS-HRM, we designed 3 primer sets to evaluate $\mathrm{CpG}$ sites 72-89. B: Dot-plot of the methylation level of $\mathrm{CpG}$ sites analyzed by MS-HRM using primers 1,2, and 3 and by cloning-based bisulfite sequencing. With primers 1 and 2 there was a strong correlation with the results of cloning-based bisulfite sequencing (primer 1: $r=0.959$; primer 2: $r=0.960$; both $p<0.01$, primer 3: $r=0.649, p=0.163$ ). C: Cloning-based bisulfite sequencing results of the MGMT promoter region in U87. Black and white indicate methylated and unmethylated CpG dinucleotides, respectively. The methylation level of each $\mathrm{CpG}$ site was calculated from the methylation status of 25 clones. The $\mathrm{CpG}$ sites analyzed by MS-HRM primers 1 (red), 2 (blue), and 3 (green) are identified at the bottom of the illustration. D: Western blots showing a strong LN18 band, a faint T98G band, and no band for the 4 other cell lines. The expression patterns were inversely correlated with the methylation levels evaluated by MS-HRM using primers 1 and 2 . Figure is available in color online only. 

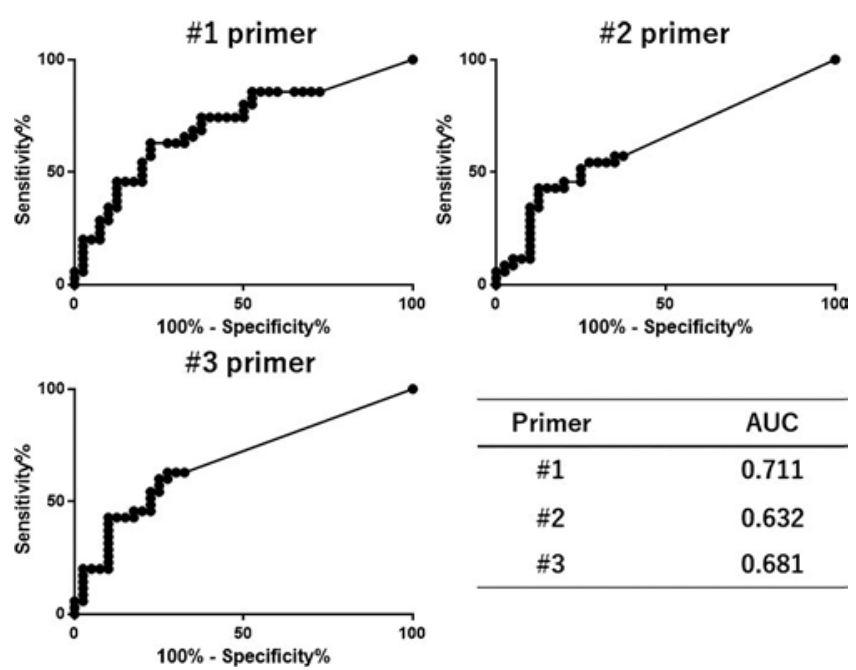

\begin{tabular}{cc}
\hline Primer & AUC \\
\hline$\# 1$ & 0.711 \\
$\# 2$ & 0.632 \\
$\# 3$ & 0.681 \\
\hline
\end{tabular}

FIG. 2. MS-HRM analysis of surgical samples. ROC analysis was applied to evaluate the sensitivity and specificity for predicting OS $\geq 18$ months. MS-HRM was with primers 1, 2, and 3 to assess the MGMT promoter methylation status. The AUC was largest with primer 1.

CpG sites; CpG 73-90 has been reported to be the critical region for transcriptional control. ${ }^{9}$ To evaluate this region by MS-HRM, we used 3 primer sets that covered CpG 72-89 (primer 1), CpG 72-83 (primer 2), and CpG 84-89 (primer 3) (Fig. 1A). ${ }^{18,21}$ For MS-PCR we applied primers commonly used for detecting the methylation status (forward primer CpG 75-78, reverse primer CpG 84-86). The primer sets for MS-HRM revealed sufficient differences in the methylation level among the 6 standard samples (methylation $0 \%, 5 \%, 10 \%, 30 \%, 50 \%$, and $100 \%$ ) to permit its evaluation in tumor cell and cell line samples (Supplemental Fig. 3).

The MGMT methylation status of the $6 \mathrm{GBM}$ cell lines was quantitatively evaluated by MS-HRM. As shown in Fig. 1B, data obtained with primers 1 and 2 revealed a similar trend; LN18 showed no methylation, T98G showed some, and the other cell lines manifested higher methylation than T98G. To validate the accuracy of these findings, we compared them with the results of cloning-based bisulfite sequencing. The methylation level of each $\mathrm{CpG}$ site was calculated from the methylation status of 25 clones. The average score of $\mathrm{CpG}$ sites covered by each primer set was recorded as the methylation level (Fig. 1C, Supplemental Figs. 4-8). Regression analysis indicated that the results of MS-HRM obtained with primers 1 and 2 exhibited a good correlation with those of cloning-based bisulfite sequencing $(r=0.959$ and $r=0.960$ for primers 1 and 2, respectively; Fig. 1B). When we compared our MS-HRM findings with the MGMT protein expression on Western blots, we found a good inverse correlation (Fig. 1D). These findings suggest that MS-HRM performed with primers 1 and 2 is useful for the quantitative evaluation of $M G M T$ promoter methylation levels.

\section{Patient Samples: MS-HRM Analysis}

$M G M T$ promoter methylation of samples from 75 GBM patients was retrospectively assessed by MS-HRM and
MS-PCR; all analyses were performed in a blinded fashion. To identify the optimal primer set for MS-HRM and the cutoff value for discriminating between the methylated and unmethylated MGMT promoter, we plotted ROC curves to predict PFS of $\geq 10$ months (Supplemental Fig. 9) and OS of $\geq 18$ months (Fig. 2). For both PFS and OS, primer 1 yielded the largest ROC AUC, suggesting that it was the most suitable for evaluating the MGMT methylation level. Calculation of the Youden index indicated that $10.65 \%$ was the optimal cutoff value for the $M G M T$ methylation level to predict PFS and OS (Supplemental Figs. 10 and 11). To validate this cutoff value, we used Kaplan-Meier estimates to compare 5 cutoff values (5\%, $8 \%, 10 \%, 12 \%$, and $15 \%$ ). The log-rank test confirmed that a cutoff value of $10 \%$ was appropriate (Table 2, Supplemental Figs. 12 and 13). Based on these findings, we used primer 1 and a cutoff value of $10 \%$ for analyzing the $M G M T$ promoter methylation status by MS-HRM.

\section{Patient Samples: Comparison of MS-HRM and MS-PCR Findings}

To evaluate the usefulness of MS-HRM for analyzing the $M G M T$ promoter methylation level, we compared MSHRM and MS-PCR findings. As shown in Fig. 3A, for the prediction of PFS and OS, MS-HRM was superior to MS-PCR. We confirmed these findings by ROC analysis. Comparison of the ROC curves depicting the sensitivity and specificity of the $M G M T$ promoter methylation status obtained by MS-HRM and MS-PCR for PFS $\geq 10$ months and $\mathrm{OS} \geq 18$ months showed that the AUC was larger with MS-HRM. This suggests that MS-HRM produces fewer false-positive and false-negative results than MS-PCR for predicting survival (Fig. 3B). Univariate and multivariate Cox regression analyses using factors such as the patient age, sex, KPS score, surgery, $I D H 1$, and the $M G M T$ methylation status determined by MS-PCR and MS-HRM $M G M T$ were performed to identify independent variables for PFS and OS. As shown in Tables 3 and 4, only data obtained by MS-HRM yielded significant prognostic indicators (MS-HRM vs MS-PCR: for PFS $\mathrm{p}=0.001$ and $\mathrm{p}=0.207$; and for OS $\mathrm{p}=0.013$ and 0.135 , respectively). Survival analysis stratified by the extent of resection also showed that MS-HRM yielded strong prognostic indicators (Supplemental Fig. 14). These findings indicate that MS-HRM was superior to MS-PCR for predicting the prognosis of patients with GBM.

To determine why MS-PCR was inferior to MS-HRM for predicting survival, we focused on the relationship between the methylation status evaluated by MS-PCR and MS-HRM using a dot-plot graph (Fig. 3C). While 3 of $31(9.7 \%)$ MS-HRM-positive samples were MS-PCR negative, more than twice as many MS-HRM-negative samples were MS-PCR positive (10 of 44, 22.8\%). The MS-PCR-positive, MS-HRM-negative samples manifested significantly higher methylation levels than MS-PCRnegative, MS-HRM-negative samples ( $\mathrm{p}<0.01$, Fig. 3C). These findings suggest that the 38 MS-PCR-positive samples were obtained with lower methylation cutoff values than the 31 MS-HRM-positive samples. In fact, when we analyzed our standard methylation samples prepared for MS-HRM by MS-PCR assay, we found that those with 5\% 
MS-HRM / PFS

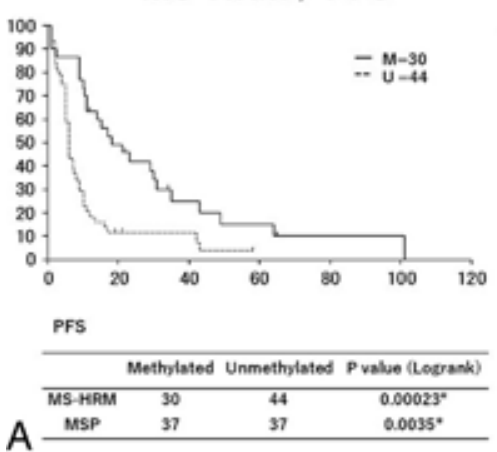

MS-HRM / OS

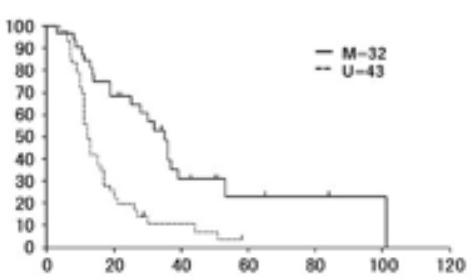

os

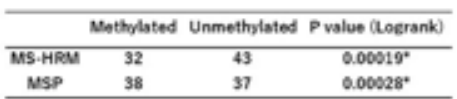

NS:

38

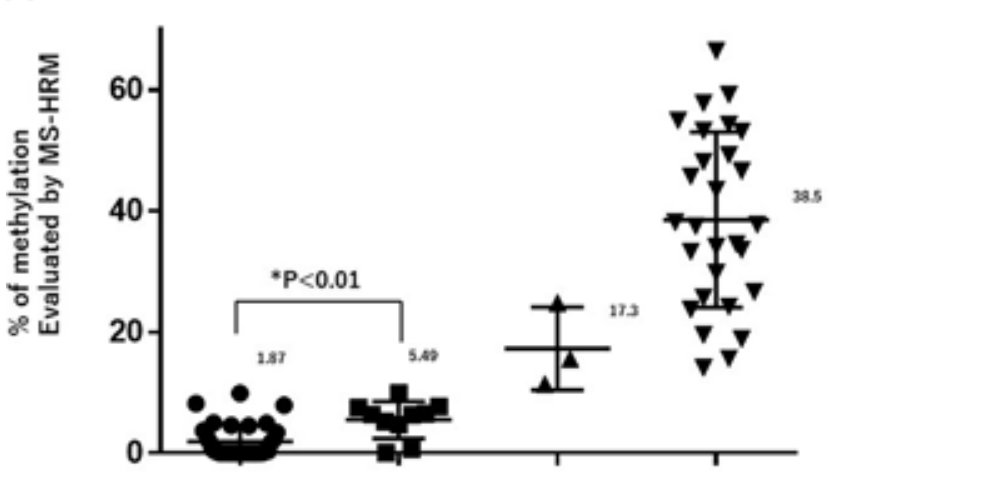

PFS

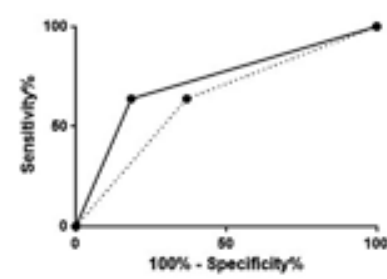

$\quad$ MSP: 0.635
B $\quad$ HRM: 0.723 os

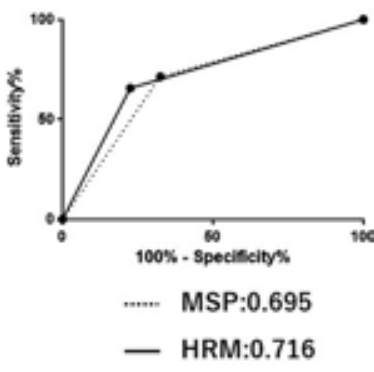

Standard samples $\begin{array}{llllll}0 \% & 5 \% & 10 \% & 30 \% & 50 \% & 100 \%\end{array}$ MS-PCR

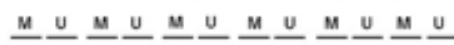

Methylation status by

MS-PCR

D MS-HRM

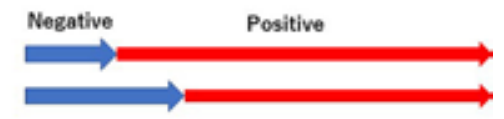

\begin{tabular}{c|ccccc} 
MS-PCR & - & + & - & + & \\
\hline MS-HRM & - & - & + & + & Cut-off : 10 \\
$C \quad(\mathrm{No})$ & $(34)$ & $(10)$ & (3) & (28) &
\end{tabular}

FIG. 3. Surgical samples. Comparison of results obtained with MS-HRM and MSP. A: Kaplan-Meier estimates of PFS and OS of 75 GBM patients based on the MGMT promoter methylation status determined by MS-HRM and MSP. Based on the log-rank test for PFS the MS-HRM and MS-PCR values were significantly different (MS-HRM: $p=0.00023$; MS-PCR: $p=0.0035$ ), as were the values for OS (MS-HRM: $p=0.00019$; MS-PCR: $p=0.00028$ ). B: ROC curves were calculated on the basis of MGMT promoter methylation determined by MS-HRM and MS-PCR (MSP) for PFS $\geq 10$ months and OS $\geq 18$ months. For both PFS and OS, the AUC was larger with MS-HRM than with MS-PCR. C: Scatterplot showing the methylation status of 75 patients classified as MS-PCR negative/MS-HRM negative $(n=34)$, MS-PCR positive/MS-HRM negative $(n=10)$, MS-PCR negative/MS-HRM positive $(n=3)$, and MS-PCR positive/MS-HMS positive $(n=28)$. Among MS-HRM-negative samples, the methylation level was significantly higher in MS-PCR-positive samples than in MS-PCR-negative samples $(p<0.01)$. D: Standard samples prepared to evaluate the methylation level by MS-HRM were analyzed by MS-PCR assay. Samples with $5 \%$ methylation featured a faint positive band, suggesting that the appropriate cutoff value for MS-PCR is $5 \% . M=$ methylated; $U=$ unmethylated. Figure is available in color online only.

methylation featured a faint positive band (Fig. 3D). This suggests that the cutoff value for MS-PCR was around 5\%. Together, our observations suggest that some methylationpositive samples identified by MS-PCR included samples with a poor prognosis and that this explains the inferiority of MS-PCR with respect to the survival of GBM patients.

\section{Discussion}

The methylation status of $M G M T$ is recognized as a strong prognostic factor in GBM patients treated with TMZ, which alkylates DNA in the O6-position of guanine. ${ }^{6,7,8}$ Moreover, studies on elderly patients with malignant astrocytoma or GBM have shown that those with MGMT promoter methylation derived similar survival benefits from TMZ monotherapy or radiotherapy alone. ${ }^{10,20}$ Consequently, information on the MGMT methylation status not only is a predictor of the patient response to TMZ, but also can be useful for selecting appropriate treatments. Although MS-PCR has been widely used to determine $M G M T$ methylation, its limitations ${ }^{9,12,23}$ led us to investigate whether MS-HRM might be a superior method.

We analyzed the special region of $M G M T$ promoter CpG sites (CpG 72-89), which Malley et al. have shown as the critical region for transcriptional control, by MSHRM with 3 primer sets. ${ }^{9}$ Our study showed that the use of primer 3, reported as the most sensitive and useful primer for detecting $M G M T$ promoter methylation in colorectal cancers by MS-HRM, exhibited a poor correlation with comparative findings made by cloning-based bisulfite sequencing. Differences in tumor types, raw-data analysis, and PCR conditions, especially differences in the annealing temperature, may account for this discrepancy. At lower annealing temperatures, the PCR primers bind both methylated and unmethylated templates, where PCR biases may favor the amplification of unmethylated 
TABLE 2. Validation of the optimal cutoff value

\begin{tabular}{cccc}
\hline & \multicolumn{2}{c}{ No. of Samples } & \\
\cline { 2 - 3 } Cutoff (\%) & Methylated & Unmethylated & p Value \\
\hline PFS & & & \\
\hline 5 & 40 & 34 & $0.0026^{*}$ \\
\hline 8 & 33 & 41 & $0.0016^{*}$ \\
\hline 10 & 30 & 44 & $0.00023^{*}$ \\
\hline 12 & 29 & 45 & $0.0092^{*}$ \\
\hline 15 & 28 & 46 & $0.00038^{*}$ \\
\hline OS & & & \\
\hline 5 & 40 & 35 & $0.00178^{*}$ \\
\hline 8 & 35 & 40 & $0.0034^{*}$ \\
\hline 10 & 31 & 44 & $0.00019^{*}$ \\
\hline 12 & 30 & 45 & $0.00025^{*}$ \\
\hline 15 & 28 & 47 & $0.0140^{*}$ \\
\hline
\end{tabular}

PFS data were missing for 1 patient. $p$ values were determined using the log-rank test.

* Statistically significant.

sequences. ${ }^{11}$ In fact, the use of primer 3 for MS-HRM assay of our 6 glioma cell lines resulted in the detection of methylation levels that were lower than those detected by cloning-based bisulfite sequencing. Optimized annealing temperatures may yield better results when primer 3 is used for MS-HRM.

To identify the best primer set, we subjected our surgical samples to ROC analysis of the MS-HRM results. The AUC was largest with primer 1 . Switzeny et al..$^{18}$ reported that MGMT promoter methylation levels determined by MS-HRM with primer 2 exhibited the best inverse correlation with $M G M T$ activity. Again, differences in instrumentation, reagents, and software used for the analysis of raw data may explain their findings, as may differences in the $\mathrm{CpG}$ sites covered by each primer. Unlike primer 1, which covered almost the full length of the region critical for transcriptional control (CpG islands 72-89), primer 2 yielded only partial coverage (CpG islands $72-83$ ). Because a pyrosequencing study by Quillien et al. ${ }^{13}$ found that the methylation level of $\mathrm{CpG} 89$ and the mean methylation level of $\mathrm{CpG} 84$ 88 were strongly correlated with the patient outcome, we think that it is advantageous to use primer 1 for evaluating critical $\mathrm{CpG}$ regions.

We found that while 5\% is the cutoff value for MSPCR, for MS-HRM the optimal cutoff is $10 \%$. According to Quillien et al., ${ }^{13}$ the cutoff value for MS-PCR is $2.5 \%$; their methylation-positive rate in GBM patients was $60 \%$ with MS-PCR and 33\% with MS-HRM. They showed that MS-HRM is superior to MS-PCR for predicting PFS, and their findings agree with ours. With respect to the cutoff values in studies predicting survival, Switzeny et al. ${ }^{18}$ identified 5\% for MS-HRM; Quillien et al. ${ }^{13}$ used 8\% for pyrosequencing. This supports the notion that the MGMT promoter region involved in transcriptional control requires some methylation levels to extend survival. Therefore, the suitable $M G M T$ methylation status for predicting survival should be determined by quantitative methods such as pyrosequencing and MS-HRM with an optimized cutoff value.

While our study documented the advantage of MSHRM over MS-PCR, MS-HRM has some disadvantages. Unlike MS-PCR, it requires a real-time PCR machine and special analysis software that may not be available in all laboratories. Moreover, there is no standardized method for estimating methylation levels with MS-HRM. We used the AUC of the normalized melting curves to generate standard curves and linear regression analysis to calculate the methylation level, as did Switzeny et al..$^{18}$ Tse et al., ${ }^{19}$ on the other hand, employed both the peak height and the AUC from normalized, temperature-shifted difference curves for generating a standard curve, ${ }^{19}$ and Wojdacz and Dobrovic ${ }^{21}$ proposed a semiquantitative estimate method based on similarities in MS-HRM patterns without a mathematical approach. Because data obtained from different real-time PCR machines may involve specific analysis methods, standardized methods for estimating MGMT methylation levels and a universal cutoff value applicable to these methods are needed for the wider acceptance of MS-HRM to predict the survival of GBM patients based on the methylation status of the MGMT promoter. We are in the process of assembling a large cohort of GBM patients to validate our current findings.

TABLE 3. Univariate and multivariate analyses of different prognostic parameters for PFS of 74 GBM patients

\begin{tabular}{|c|c|c|c|c|c|c|}
\hline \multirow[b]{2}{*}{ Variable } & \multicolumn{3}{|c|}{ Univariate } & \multicolumn{3}{|c|}{ Multivariate } \\
\hline & $\mathrm{p}$ Value & $\mathrm{HR}$ & $95 \% \mathrm{Cl}$ & $p$ Value & $\mathrm{HR}$ & $95 \% \mathrm{Cl}$ \\
\hline Age (<65 vs $\geq 65$ yrs) & 0.129 & 0.702 & $0.399-1.125$ & & & \\
\hline Sex (F vs M) & 0.549 & 1.153 & $0.695-1.982$ & & & \\
\hline KPS score $(<80$ vs $\geq 80)$ & $0.026^{*}$ & 0.591 & $0.333-0.934$ & 0.588 & 0.847 & $0.465-1.543$ \\
\hline Surgery (GTR vs other) & $<0.001^{*}$ & 0.372 & $0.123-0.450$ & $<0.001^{*}$ & 0.237 & $0.126-0.444$ \\
\hline IDH1 (mutated vs wild type) & 0.159 & 0.458 & $0.228-1.274$ & & & \\
\hline MS-PCR MGMT (methylated vs unmethylated) & $0.0035^{*}$ & 0.519 & $0.259-0.767$ & 0.207 & 0.671 & $0.324-1.163$ \\
\hline MS-HRM MGMT (methylated vs unmethylated) & $0.00023^{*}$ & 0.432 & $0.217-0.627$ & $0.001^{*}$ & 0.349 & $0.215-0.832$ \\
\hline
\end{tabular}


TABLE 4. Univariate and multivariate analyses of different prognostic parameters for OS of 75 GBM patients

\begin{tabular}{|c|c|c|c|c|c|c|}
\hline \multirow[b]{2}{*}{ Variable } & \multicolumn{3}{|c|}{ Univariate } & \multicolumn{3}{|c|}{ Multivariate } \\
\hline & p Value & $\mathrm{HR}$ & $95 \% \mathrm{Cl}$ & $p$ Value & $\mathrm{HR}$ & $95 \% \mathrm{Cl}$ \\
\hline Age (<65 vs $\geq 65$ yrs) & 0.065 & 0.636 & $0.345-1.032$ & & & \\
\hline Sex (F vs M) & 0.254 & 1.334 & $0.798-2.350$ & & & \\
\hline KPS score (<80 vs $\geq 80)$ & 0.641 & 0.888 & $0.520-1.495$ & & & \\
\hline Surgery (GTR vs other) & $<0.001^{*}$ & 0.103 & $0.002-0.013$ & $<0.001^{*}$ & 0.363 & $0.208-0.633$ \\
\hline IDH1 (mutated vs wild type) & 0.059 & 0.288 & $0.192-1.031$ & & & \\
\hline MS-PCR MGMT (methylated vs unmethylated) & $0.00028^{*}$ & 0.424 & $0.198-0.616$ & 0.135 & 0.614 & $0.324-1.163$ \\
\hline MS-HRM MGMT (methylated vs unmethylated) & $0.00019^{*}$ & 0.399 & $0.207-0.613$ & $0.013^{*}$ & 0.423 & $0.215-0.832$ \\
\hline
\end{tabular}

* Statistically significant.

\section{Conclusions}

Our study showed that MS-HRM is superior to MSPCR for the identification of GBM patients likely to respond to TMZ treatment. As its results are reliable, we suggest MS-HRM as an alternative to MS-PCR for assessing the prognosis of patients with GBM.

\section{Acknowledgments}

We thank Ms. Ayumi Nagatomo and Ms. I. Asya for their technical assistance and Ms. U. Petralia for her editorial assistance.

\section{References}

1. Costello JF, Futscher BW, Kroes RA, Pieper RO: Methylation-related chromatin structure is associated with exclusion of transcription factors from and suppressed expression of the O-6-methylguanine DNA methyltransferase gene in human glioma cell lines. Mol Cell Biol 14:6515-6521, 1994

2. Costello JF, Futscher BW, Tano K, Graunke DM, Pieper RO: Graded methylation in the promoter and body of the $\mathrm{O}^{6}$ methylguanine DNA methyltransferase (MGMT) gene correlates with MGMT expression in human glioma cells. J Biol Chem 269:17228-17237, 1994

3. Dunn J, Baborie A, Alam F, Joyce K, Moxham M, Sibson R, et al: Extent of MGMT promoter methylation correlates with outcome in glioblastomas given temozolomide and radiotherapy. Br J Cancer 101:124-131, 2009

4. Esteller M, Garcia-Foncillas J, Andion E, Goodman SN, Hidalgo OF, Vanaclocha V, et al: Inactivation of the DNA-repair gene MGMT and the clinical response of gliomas to alkylating agents. N Engl J Med 343:1350-1354, 2000

5. Esteller M, Hamilton SR, Burger PC, Baylin SB, Herman JG: Inactivation of the DNA repair gene $\mathrm{O}^{6}$-methylguanineDNA methyltransferase by promoter hypermethylation is a common event in primary human neoplasia. Cancer Res 59:793-797, 1999

6. Hegi ME, Diserens AC, Godard S, Dietrich PY, Regli L, Ostermann S, et al: Clinical trial substantiates the predictive value of O-6-methylguanine-DNA methyltransferase promoter methylation in glioblastoma patients treated with temozolomide. Clin Cancer Res 10:1871-1874, 2004

7. Hegi ME, Diserens AC, Gorlia T, Hamou MF, de Tribolet N, Weller M, et al: MGMT gene silencing and benefit from temozolomide in glioblastoma. N Engl J Med 352:997-1003, 2005

8. Kaina B, Christmann M, Naumann S, Roos WP: MGMT: key node in the battle against genotoxicity, carcinogenicity and apoptosis induced by alkylating agents. DNA Repair (Amst) 6:1079-1099, 2007

9. Malley DS, Hamoudi RA, Kocialkowski S, Pearson DM,
Collins VP, Ichimura K: A distinct region of the MGMT $\mathrm{CpG}$ island critical for transcriptional regulation is preferentially methylated in glioblastoma cells and xenografts. Acta Neuropathol 121:651-661, 2011

10. Malmström A, Grønberg BH, Marosi C, Stupp R, Frappaz D, Schultz H, et al: Temozolomide versus standard 6-week radiotherapy versus hypofractionated radiotherapy in patients older than 60 years with glioblastoma: the Nordic randomised, phase 3 trial. Lancet Oncol 13:916-926, 2012

11. Mikeska T, Bock C, El-Maarri O, Hübner A, Ehrentraut D, Schramm J, et al: Optimization of quantitative MGMT promoter methylation analysis using pyrosequencing and combined bisulfite restriction analysis. J Mol Diagn 9:368-381, 2007

12. Preusser M, Elezi L, Hainfellner JA: Reliability and reproducibility of PCR-based testing of O6-methylguanine-DNA methyltransferase gene (MGMT) promoter methylation status in formalin-fixed and paraffin-embedded neurosurgical biopsy specimens. Clin Neuropathol 27:388-390, 2008

13. Quillien V, Lavenu A, Karayan-Tapon L, Carpentier C, Labussière M, Lesimple T, et al: Comparative assessment of 5 methods (methylation-specific polymerase chain reaction, MethyLight, pyrosequencing, methylation-sensitive highresolution melting, and immunohistochemistry) to analyze O6-methylguanine-DNA-methyltranferase in a series of 100 glioblastoma patients. Cancer 118:4201-4211, 2012

14. Ricard D, Idbaih A, Ducray F, Lahutte M, Hoang-Xuan K, Delattre JY: Primary brain tumours in adults. Lancet 379:1984-1996, 2012

15. Saito K, Hirai T, Takeshima H, Kadota Y, Yamashita S, Ivanova A, et al: Genetic factors affecting intraoperative 5-aminolevulinic acid-induced fluorescence of diffuse gliomas. Radiol Oncol 51:142-150, 2017

16. Stupp R, Hegi ME, Mason WP, van den Bent MJ, Taphoorn MJ, Janzer RC, et al: Effects of radiotherapy with concomitant and adjuvant temozolomide versus radiotherapy alone on survival in glioblastoma in a randomised phase III study: 5-year analysis of the EORTC-NCIC trial. Lancet Oncol 10:459-466, 2009

17. Stupp R, Mason WP, van den Bent MJ, Weller M, Fisher $\mathrm{B}$, Taphoorn MJ, et al: Radiotherapy plus concomitant and adjuvant temozolomide for glioblastoma. N Engl J Med 352:987-996, 2005

18. Switzeny OJ, Christmann M, Renovanz M, Giese A, Sommer C, Kaina B: MGMT promoter methylation determined by HRM in comparison to MSP and pyrosequencing for predicting high-grade glioma response. Clin Epigenetics 8:49, 2016

19. Tse MY, Ashbury JE, Zwingerman N, King WD, Taylor SA, Pang SC: A refined, rapid and reproducible high resolution melt (HRM)-based method suitable for quantification of global LINE-1 repetitive element methylation. BMC Res Notes 4:565, 2011 
20. Wick W, Platten M, Meisner C, Felsberg J, Tabatabai G, Simon M, et al: Temozolomide chemotherapy alone versus radiotherapy alone for malignant astrocytoma in the elderly: the NOA-08 randomised, phase 3 trial. Lancet Oncol 13:707-715, 2012

21. Wojdacz TK, Dobrovic A: Methylation-sensitive high resolution melting (MS-HRM): a new approach for sensitive and high-throughput assessment of methylation. Nucleic Acids Res 35:e41, 2007

22. Xu-Welliver M, Pegg AE: Degradation of the alkylated form of the DNA repair protein, $\mathrm{O}^{6}$-alkylguanine-DNA alkyltransferase. Carcinogenesis 23:823-830, 2002

23. Yang SH, Lee KS, Yang HJ, Jeon BH, Lee YS, Nam SW, et al: $\mathrm{O}^{6}$-methylguanine-DNA-methyltransferase promoter methylation assessment by microdissection-assisted methylation-specific PCR and high resolution melting analysis in patients with glioblastomas. J Neurooncol 106:243-250, 2012

\section{Disclosures}

The authors report no conflict of interest concerning the materi- als or methods used in this study or the findings specified in this paper.

\section{Author Contributions}

Conception and design: Yamashita, Yokogami. Acquisition of data: Yamashita, Matsumoto, Saito, Mizuguchi. Analysis and interpretation of data: Yamashita, Yokogami. Drafting the article: Yokogami, Takeshima. Reviewed submitted version of manuscript: Yamashita, Yokogami, Takeshima. Approved the final version of the manuscript on behalf of all authors: Yamashita. Statistical analysis: Yamashita. Study supervision: Yokogami, Ohta, Takeshima.

\section{Online-Only Content}

Supplemental material is available with the online version of the article.

Supplemental Figs. 1-14. https://thejns.org/doi/suppl/10.3171/ 2017.11.JNS171710.

\section{Correspondence}

Shinji Yamashita: University of Miyazaki, Japan. shinjy@med. miyazaki-u.ac.jp. 